\title{
A DISCRETE-CONTINUOUS METHOD OF MECHANICAL SYSTEM MODELLING
}

\author{
Rafał Hein
}

Gdansk University of Technology,Poland

\begin{abstract}
The paper describes a discrete-continuous method of dynamic system modelling. The presented approach is hybrid in its nature, as it combines the advantages of spatial discretization methods with those of continuous system modelling methods. In the proposed method, a three-dimensional system is discretised in two directions only, with the third direction remaining continuous. The thus obtained discrete-continuous model is described by a set of coupled partial differential equations, derived using the rigid finite element method (RFEM). For this purpose, firstly the general differential equations are written. Then these equations are converted into difference equations. The derived equations, expressed in matrix form, allow to create a global matrix for the whole system. They are solved using the distributed transfer function method. The proposed approach is illustrated with the examples of a simple beam fixed at both ends and a simply supported plate.
\end{abstract}

Keywords: modelling, mechanical system, dynamic systems, vibrations, hybrid modelling methods.

\section{INTRODUCTION}

Continuous systems with spatially distributed parameters are described by partial differential equations. However, exact solutions to such equations exist only for a small class of simple technical problems. For this reason, the continuous structure is usually discretized and divided into small, spatially lumped parameter elements. Such elements are called finite elements and the method is well known as the finite element method. A discrete model obtained in this way is described by the set of ordinary differential equations. The solutions to such equations pose no problem but the model is approximate and its accuracy depends on the number of finite elements. The greater their number, the more accurate the model. However, there is an optimal division density, above which the rounding errors start to seriously affect the numerical calculation. Additionally, a very large number of finite elements increases the order of the differential equation, which is not convenient when designing, for instance, control systems. Such a model also requires considerable computer run time and huge memory capacity to obtain and store numerical calculation results.

In the case of slender elements, such as: strings, bars and beams, for which the analytical and exact solutions are well known, it is better to use an accurate, continuous model description.

Consequently, there is no universal approach which is both accurate and applicable to a wide range of dynamic systems. That is why the modelling methods of physical systems are constantly being developed and improved.

The paper proposes a hybrid method of modelling that combines the advantages of the spatial discretization methods with those of continuous system modelling methods.

In the classical finite element method, the body is discretized in all three spatial directions (Fig. 1a). In the proposed method, the same body is discretized only in two spatial directions (Fig. 1b), with one direction remaining continuous. Such a division results in the appearance of discrete-continuous elements, so-called prisms (Fig. 1b), with 
parameters distributed along one of the axes. Consequently, the obtained elements are one-dimensional distributed elements, described by second-order partial differential equations. However, these equations also include terms related to interactions between elements, as a result of which the entire system is described by coupled second-order partial differential equations.

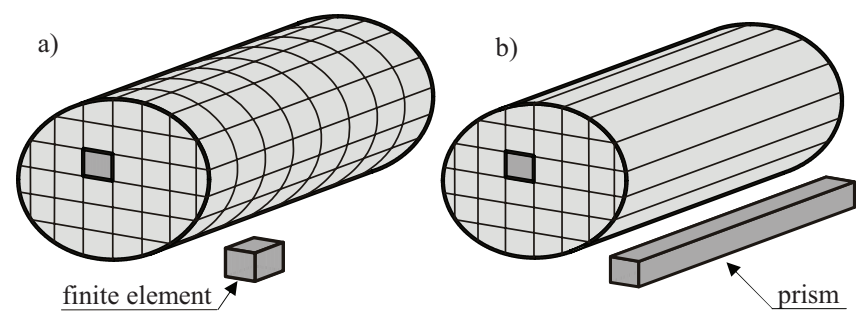

Fig. 1. Spatial discretization of $3 D$ body: a) conventional finite element method, b) proposed hybrid method

The obtained equations are solved by using the distributed transfer function method $[15,16,18]$. This method enables to obtain analytical or semi-analytical solutions for $1 \mathrm{D}$ and 3D systems, respectively. A similar idea was considered in [12] and [15]. However, in some aspects there are fundamental differences. In [12] and [18] the body is treated as deformable in the directions in which the discretization was made, while in the proposed method it is treated as a rigid and non-deformable solid in two discretization directions. This approach has many advantages. Firstly, it makes it possible to obtain a simple and clear system of partial differential equations. Then, by using the modal decomposition method, it is easy to determine the modal model and analyse it in the time domain $[1,9,10,11]$. In a simple way, the modal model can be reduced to a low order model, which is convenient in design and analysis of control systems $[2,3,4]$. The proposed method has been applied in modelling $1 \mathrm{D}, 2 \mathrm{D}$ and $3 \mathrm{D}$ systems $[8,10,12,17]$ and may be used to modelling and solving many practical engineering problems $[6,7]$.

\section{GENERAL MODEL OF THE DISCRETE- CONTINUOUS SYSTEM}

In order to derive a general model of the discrete-continuous system, let us consider two prisms, $r$ and $p$, connected by a spring-damping layer, $k$, continuous in the $x$ direction, with distributed parameters (Fig. 2a). The discretized model of the analysed system is shown in Fig. 5. Each element has 6 degrees of freedom expressed by means of general displacements. The first three of them are translational displacements along the $x_{1}, x_{2}$, and $x_{3}$-axes, while the remaining represent rotational displacements around these axes:

$$
\begin{gathered}
\boldsymbol{q}_{r-1}=\operatorname{col}\left(q_{r-1,1}, q_{r-1,2}, q_{r-1,3}, q_{r-1,4}, q_{r-1,5}, q_{r-1,6}\right), \\
\boldsymbol{q}_{r}=\operatorname{col}\left(q_{r, 1}, q_{r, 2}, q_{r, 3}, q_{r, 4}, q_{r, 5}, q_{r, 6}\right)
\end{gathered}
$$

$$
\boldsymbol{q}_{r+1}=\operatorname{col}\left(q_{r+1,1}, q_{r+1,2}, q_{r+1,3}, q_{r+1,4}, q_{r+1,5}, q_{r+1,6}\right),
$$

$$
\boldsymbol{q}_{p}=\operatorname{col}\left(q_{p, 1}, q_{p, 2}, q_{p, 3}, q_{p, 4}, q_{p, 5}, q_{p, 6}\right),
$$

a)

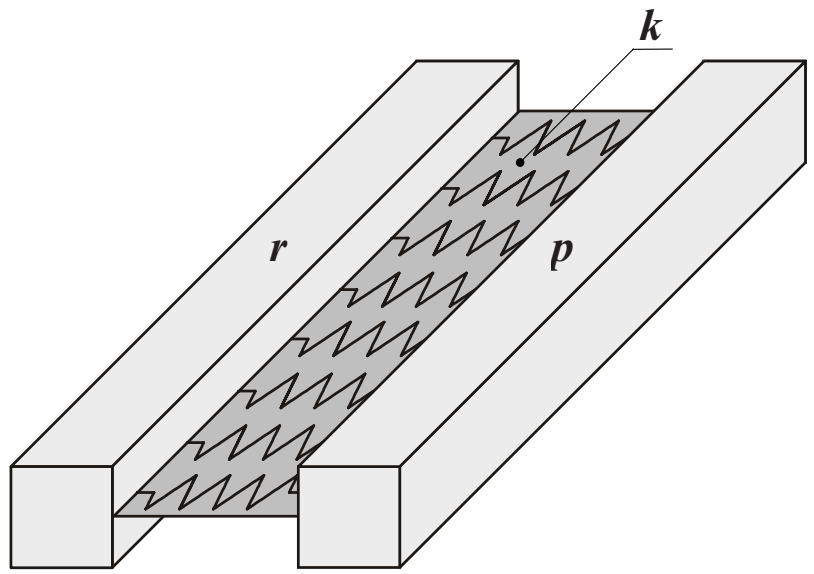

b)

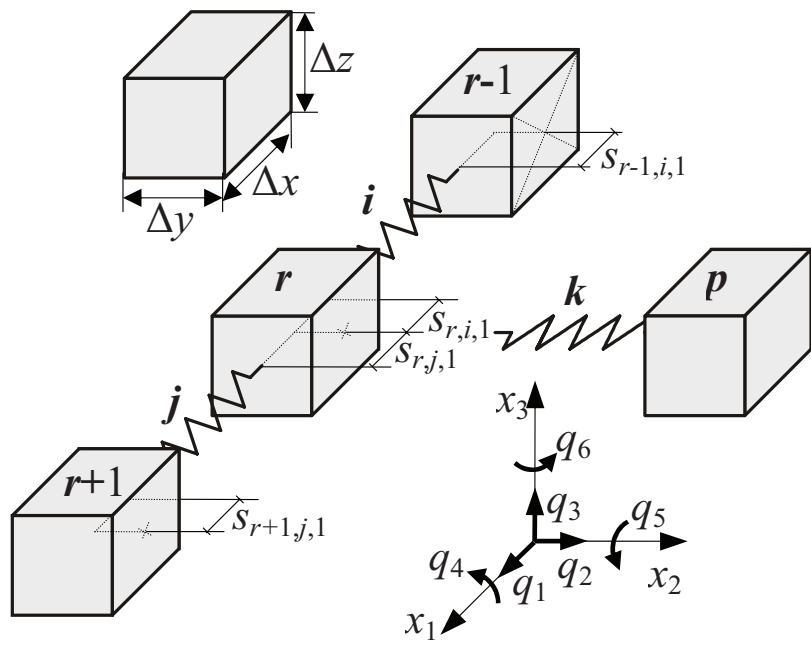

Fig. 2. General model of the analysed system a) discrete-continuous, b) discrete

For the $r$-th rigid element of the discrete model presented in Fig. 2b, a system of ordinary differential equations was written and verified by using the rigid finite element method $[5,13,14]$. The obtained equations have the following form:

$$
\begin{aligned}
& f_{r, 1}=m \ddot{q}_{r, 1}+c_{i, 1}\left(q_{r, 1}-q_{r-1,1}\right)-c_{j, 1}\left(q_{r+1,1}-q_{r, 1}\right)+ \\
& \quad+c_{k, 1}\left(q_{r, 1}-q_{p, 1}\right)+c_{k, 1}\left(s_{r, k, 3} q_{r, 5}-s_{p, k, 3} q_{p, 5}\right)+ \\
& \quad-c_{k, 1}\left(s_{r, k, 2} q_{r, 6}-s_{p, k, 2} q_{p, 6}\right)+ \\
& \quad+c_{i, 1}\left(s_{r, i, 3} q_{r, 5}-s_{r-1, i, 3} q_{r-1,5}\right)-c_{i, 1}\left(s_{r, i, 2} q_{r, 6}-s_{r-1, i, 2} q_{r-1,6}\right)+ \\
& \quad+c_{j, 1}\left(s_{r, j, 3} q_{r, 5}-s_{p, j, 3} q_{r+1,5}\right)-c_{j, 1}\left(s_{r, j, 2} q_{r, 6}-s_{p, j, 2} q_{r+1,6}\right), \\
& f_{r, 2}=m \ddot{q}_{r, 2}+c_{i, 2}\left(q_{r, 2}-q_{i-1, j, 2}\right)-c_{j, 2}\left(q_{r+1,2}-q_{r, 2}\right)+ \\
& \quad+c_{k, 2}\left(q_{r, 2}-q_{p, 2}\right)-c_{k, 2}\left(s_{r, k, 3} q_{r, 4}-s_{p, k, 3} q_{p, 4}\right)+ \\
& \quad+c_{k, 2}\left(s_{r, k, 1} q_{r, 6}-s_{p, k, 1} q_{p, 6}\right)+ \\
& \quad+c_{i, 2}\left(s_{r, i, 1} q_{r, 6}-s_{r-1, i, 1} q_{r-1,6}\right)+c_{j, 2}\left(s_{r, j, 1} q_{r, 6}-s_{p, j, 1} q_{r+1,6}\right)+ \\
& \quad+c_{j, 2}\left(s_{p, j, 3} q_{r+1,4}-s_{r, j, 3} q_{r, 4}\right)+c_{i, 2}\left(s_{r-1, i, 3} q_{r-1,4}-s_{r, i, 3} q_{r, 4}\right),
\end{aligned}
$$




$$
\begin{aligned}
& f_{r, 3}=m \ddot{q}_{r, 3}+c_{i, 3}\left(q_{r, 3}-q_{r-1,3}\right)-c_{j, 3}\left(q_{r+1,3}-q_{r, 3}\right)+ \\
& +c_{k, 3}\left(q_{r, 3}-q_{p, 3}\right)+c_{k, 3}\left(s_{r, k, 2} q_{r, 4}-s_{p, k, 2} q_{p, 4}\right)+ \\
& +c_{p, 3}\left(s_{p, k, 1} q_{p, 5}-s_{r, k, 1} q_{r, 5}\right)+ \\
& -c_{i, 3}\left(s_{r, i, 1} q_{r, 5}-s_{r-1, i, 1} q_{r-1,5}\right)-c_{j, 3}\left(s_{r, j, 1} q_{r, 5}-s_{r+1, j, 1} q_{r+1,5}\right)+ \\
& +c_{i, 3}\left(s_{r, i, 2} q_{r, 4}-s_{r-1, i, 2} q_{r-1,4}\right)+c_{j, 3}\left(s_{r, j, 2} q_{r, 4}-s_{r+1, j, 2} q_{r+1,4}\right) \text {, } \\
& f_{r, 4}=\rho \Delta x I_{0 x} \ddot{q}_{r, 4}+c_{i, 4}\left(q_{r, 4}-q_{r-1,4}\right)-c_{j, 4}\left(q_{r+1,4}-q_{r, 4}\right)+ \\
& +c_{k, 4}\left(q_{r, 4}-q_{p, 4}\right)+c_{k, 3}\left(s_{r, k, 2} q_{r, 3}-s_{r, k, 2} q_{p, 3}\right)+ \\
& +c_{k, 3}\left(s_{r, k, 2}^{2} q_{r, 4}-s_{r, k, 2} s_{p, k, 2} q_{p, 4}\right)+ \\
& +c_{k, 2}\left(s_{r, k, 3}^{2} q_{r, 4}-s_{r, k, 3} s_{p, k, 3} q_{p, 4}\right)+c_{k, 2}\left(s_{r, k, 3} q_{p, 2}-s_{r, k, 3} q_{r, 2}\right)+ \\
& -c_{k, 2}\left(s_{r, k, 1} s_{r, k, 3} q_{r, 6}-s_{r, k, 3} s_{p, k, 1} q_{p, 6}\right)+ \\
& -c_{k, 3}\left(s_{r, k, 1} s_{r, k, 2} q_{r, 5}-s_{r, k, 2} s_{p, k, 1} q_{p, 5}\right)+ \\
& -c_{i, 2}\left(s_{r, i, 3} q_{r, 2}-s_{r, i, 3} q_{r-1,2}\right)-c_{j, 2}\left(s_{r, j, 3} q_{r, 2}-s_{r, j, 3} q_{r+1,2}\right)+ \\
& +c_{i, 3}\left(s_{r, i, 2} q_{r, 3}-s_{r, i, 2} q_{r-1,3}\right)+c_{j, 3}\left(s_{r, j, 2} q_{r, 3}-s_{r, j, 2} q_{r+1,3}\right)+ \\
& +c_{i, 3}\left(s_{r-1, i, 1} s_{r, i, 2} q_{r-1,5}-s_{r, i, 1} s_{r, i, 2} q_{r, 5}\right)+ \\
& +c_{j, 3}\left(s_{r, j, 2} s_{r+1, j, 1} q_{r+1,5}-s_{r, j, 1} s_{r, j, 2} q_{r, 5}\right)+ \\
& +c_{i, 3}\left(s_{r, i, 2}^{2} q_{r, 4}-s_{r-1, i, 2} s_{r, i, 2} q_{r-1,4}\right)+ \\
& +c_{i, 2}\left(s_{r, i, 3}^{2} q_{r, 4}-s_{r-1, i, 3} s_{r, i, 3} q_{r-1,4}\right)+ \\
& +c_{j, 3}\left(s_{r, j, 2}^{2} q_{i, j, k, 4}-s_{r, j, 2} s_{r+1, j, 2} q_{r-1,4}\right)+ \\
& +c_{j, 2}\left(s_{r, j, 3}^{2} q_{r, 4}-s_{r, j, 3} s_{r+1, j, 3} q_{r+1,4}\right)+ \\
& +c_{i, 2}\left(s_{r-1, i, 1} s_{r, i, 3} q_{r-1,6}-s_{r, i, 1} s_{r, i, 3} q_{r, 6}\right)+ \\
& +c_{j, 2}\left(s_{r, j, 3} s_{r+1, j, 1} q_{r+1,6}-s_{r, j, 1} s_{r, j, 3} q_{r, 6}\right) \text {, } \\
& f_{r, 5}=\rho \Delta y I_{0 y} \ddot{q}_{r, 5}+c_{i, 5}\left(q_{r, 5}-q_{r-1,5}\right)-c_{j, 5}\left(q_{r+1,5}-q_{r, 5}\right)+ \\
& +c_{k, 5}\left(q_{r, 5}-q_{p, 5}\right)-c_{i, 3} s_{r, i, 1}\left(q_{r, 3}-q_{r-1,3}\right)-c_{k, 3} s_{r, k, 1}\left(q_{r, 3}-q_{p, 3}\right) \\
& +c_{i, 3}\left(s_{r, i, 1}^{2} q_{r, 5}-s_{r-1, i, 1} s_{r, i, 1} q_{r-1,5}\right)+ \\
& +c_{j, 3}\left(s_{r, j, 1}^{2} q_{r, 5}-s_{r, j, 1} s_{r+1, j, 1} q_{r+1,5}\right)+ \\
& +c_{k, 1} s_{r, k, 3}\left(q_{r, 1}-q_{p, 1}\right)+c_{k, 3}\left(s_{r, k, 1}^{2} q_{r, 5}-s_{r, k, 1} s_{p, k, 1} q_{p, 5}\right)+ \\
& +c_{k, 1}\left(s_{r, k, 3}^{2} q_{r, 5}-s_{r, k, 3} s_{p, k, 3} q_{p, 5}\right)+c_{j, 3} s_{r, j, 1}\left(q_{r+1,3}-q_{r, 3}\right)+ \\
& -c_{k, 3}\left(s_{r, k, 1} s_{r, k, 2} q_{r, 4}-s_{r, k, 1} s_{p, k, 2} q_{p, 4}\right)+ \\
& -c_{k, 1}\left(s_{r, k, 2} s_{r, k, 3} q_{r, 6}-s_{r, k, 3} s_{p, k, 2} q_{p, 6}\right)+ \\
& +c_{i, 1} s_{r, i, 3}\left(q_{r, 1}-q_{r-1,1}\right)+c_{j, 1} s_{r, j, 3}\left(q_{r, 1}-q_{r+1,1}\right)+ \\
& +c_{i, 1}\left(s_{r, i, 3}^{2} q_{r, 5}-s_{r-1, i, 3} s_{r, i, 3} q_{r-1,5}\right)+ \\
& +c_{j, 1}\left(s_{r, j, 3}^{2} q_{r, 5}-s_{r, j, 3} s_{r+1, j, 3} q_{r+1,5}\right)+ \\
& +c_{i, 3}\left(s_{r-1, i, 2} s_{r, i, 1} q_{r-1,4}-s_{r, i, 2} s_{r, i, 1} q_{r, 4}\right)+ \\
& +c_{j, 3}\left(s_{r, j, 1} s_{r+1, j, 2} q_{r+1,4}-s_{r, j, 1} s_{r, j, 2} q_{r, 4}\right)+ \\
& +c_{i, 1}\left(s_{r-1, i, 2} s_{r, i, 3} q_{r-1,6}-s_{r, i, 2} s_{r, i, 3} q_{r, 6}\right)+ \\
& +c_{j, 1}\left(s_{r+1, j, 2} s_{r, j, 3} q_{r+1,6}-s_{r, j, 2} s_{r, j, 3} q_{r, 6}\right),
\end{aligned}
$$

$$
\begin{aligned}
& f_{r, 6}=\rho \Delta z I_{0 z} \ddot{q}_{r, 6}+c_{i, 6}\left(q_{r, 6}-q_{r-1,6}\right)-c_{j, 6}\left(q_{r+1,6}-q_{r, 6}\right)+ \\
& \quad+c_{j, 2} s_{r, j, 1}\left(q_{r, 2}-q_{r+1,2}\right)-c_{i, 2} s_{r, i, 1}\left(q_{r-1,2}-q_{r, 2}\right)+ \\
& \quad+c_{k, 6}\left(q_{r, 6}-q_{p, 6}\right)-c_{k, 1} s_{r, k, 2}\left(q_{r, 1}-q_{p, 1}\right)+ \\
& \quad-c_{k, 1}\left(s_{r, k, 2} s_{r, k, 3} q_{r, 5}-s_{r, k, 2} s_{p, k, 3} q_{p, 5}\right) \\
& \quad+c_{i, 2}\left(s_{r, i, 1}^{2} q_{r, 6}-s_{r-1, i, 1} s_{r, i, 1} q_{r-1,6}\right)+ \\
& \quad+c_{j, 2}\left(s_{r, j, 1}^{2} q_{r, 6}-s_{r, j, 1} s_{r+1, j, 1} q_{r+1,6}\right)+ \\
& \quad+c_{k, 1}\left(s_{r, k, 2}^{2} q_{r, 6}-s_{r, k, 2} s_{p, k, 2} q_{p, 6}\right)+ \\
& \quad+c_{k, 2} s_{r, k, 1}\left(q_{r, 2}-q_{p, 2}\right)- \\
& \quad-c_{k, 2}\left(s_{r, k, 1} s_{r, k, 3} q_{r, 4}-s_{r, k, 1} s_{p, k, 3} q_{p, 4}\right)+ \\
& \quad+c_{k, 2}\left(s_{r, k, 1}^{2} q_{r, 6}-s_{r, k, 1} s_{p, k, 1} q_{p, 6}\right)- \\
& \quad-c_{i, 1} s_{r, i, 2}\left(q_{r, 1}-q_{r-1,1}\right)+c_{j, 1} s_{r, j, 2}\left(q_{r+1,1}-q_{r, 1}\right)+ \\
& \quad+c_{i, 1}\left(s_{r-1, i, 3} s_{r, i, 2} q_{r-1,5}-s_{r, i, 2} s_{r, i, 3} q_{r, 5}\right)+ \\
& \quad+c_{j, 1}\left(s_{r, j, 2} s_{r+1, j, 3} q_{r+1,5}-s_{r, j, 2} s_{r, j, 3} q_{r, 5}\right)+ \\
& \quad+c_{i, 2}\left(s_{r-1, i, 3} s_{r, i, 1} q_{r-1,4}-s_{r, i, 1} s_{r, i, 3} q_{r, 4}\right)+ \\
& \quad+c_{j, 2}\left(s_{r, j, 1} s_{r+1, j, 3} q_{r+1,4}-s_{r, j, 1} s_{r, j, 3} q_{r, 4}\right)+ \\
& +c_{i, 1}\left(s_{r, i, 2}^{2} q_{r, 6}-s_{r-1, i, 2} s_{r, i, 2} q_{r-1,6}\right)+ \\
& +c_{j, 1}\left(s_{r, j, 2}^{2} q_{r, 6}-s_{r, j, 2} s_{r+1, j, 2} q_{r+1,6}\right),
\end{aligned}
$$

where: $f_{r, \alpha}$ is the general force applied to the $r$-th element (excitation) in the $x_{\alpha}$-axis direction (Fig. $2 \mathrm{~b}$ ); $m$ is the mass of the rigid finite element; $\rho$ is the density of the rigid element; $I_{0 \alpha}$ is the geometric, polar moment of inertia around the $x_{\alpha}$-axis; $\Delta x, \Delta y, \Delta z$ are the elementary dimensions of the finite element (Fig. 2b); $q_{r \alpha}$ is the general displacement of the $r$-th element in the $x_{\alpha}$-axis direction; $q_{p, \alpha}$ is the general displacement of the $p$-th element in the $x_{\alpha}$-axis direction; $s_{\alpha, \beta, \gamma}$ is the distance between the body $\alpha$ and the spring-damping element $\beta$ in the $x_{\gamma}$-axis direction, and $c_{\alpha, \beta}$ is the stiffness coefficient of the spring element $\alpha$ in the $x_{\beta}$ direction $[5,13,14]$ :

$$
\begin{aligned}
& c_{i, 1}=c_{j, 1}=\frac{E \Delta y \Delta z}{\Delta x}, c_{k, 1}=\frac{\kappa G \Delta x \Delta z}{\Delta y}, \\
& c_{i, 2}=c_{j, 2}=\frac{\kappa G \Delta y \Delta z}{\Delta x}, c_{k, 2}=\frac{E \Delta x \Delta z}{\Delta y}, \\
& c_{i, 3}=c_{j, 3}=\frac{\kappa G \Delta y \Delta z}{\Delta x}, c_{k, 3}=\frac{\kappa G \Delta x \Delta z}{\Delta y}, \\
& c_{i, 4}=c_{j, 4}=\frac{G I_{0 x}}{\Delta x}, c_{k, 4}=\frac{E I_{x y}}{\Delta y}, \\
& c_{i, 5}=c_{j, 5}=\frac{E I_{y x}}{\Delta x}, c_{k, 5}=\frac{G I_{0 y}}{\Delta y}, \\
& c_{i, 6}=c_{j, 6}=\frac{E I_{z x}}{\Delta x}, c_{k, 6}=\frac{E I_{z y}}{\Delta y},
\end{aligned}
$$

In Eq. (11): $E$ is the Young's modulus, $G$ is the Kirchhoff shear modulus, $I_{\alpha, \beta}$ is the geometric moment of inertia of the cross-section area perpendicular to the $x_{\beta}$-axis around the $x_{\alpha}$-axis, and $\kappa$ is the numerical shape factor of the cross section area.

The rigid finite element model described by Equations $(5) \div(10)$ is next transformed to a discrete-continuous model. We assume that the discretization is not performed in the $x$ direction. By putting: 


$$
\begin{aligned}
& s_{r-1, i, 1}=s_{r, j, 1}=\frac{\Delta x}{2}, s_{r, i, 1}=s_{r+1, j, 1}=-\frac{\Delta x}{2}, \\
& s_{r, i, 2}=s_{r, i, 3}=s_{r, j, 2}=s_{r, j, 3}=s_{r+1, j, 2}=s_{r+1, j, 3}=s_{r, k, 1}=s_{p, k, 1}=0
\end{aligned}
$$

and (11) into Eqs. (5) $\div(10)$, and dividing these equations by $\Delta x$ we obtain the following formulas:

$$
\begin{aligned}
& f_{x r, 1}=\rho \Delta y \Delta z \ddot{q}_{r, 1}-E \Delta y \Delta z \frac{\frac{q_{r+1,1}-q_{r, 1}}{\Delta x}-\frac{q_{r, 1}-q_{r-1,1}}{\Delta x}}{\Delta x}+ \\
& +\frac{\kappa G \Delta z}{\Delta y}\left(q_{r, 1}-q_{p, 1}\right)+\frac{\kappa G \Delta z}{\Delta y}\left(s_{r, k, 3} q_{r, 5}-s_{p, k, 3} q_{p, 5}\right)+ \\
& -\frac{\kappa G \Delta z}{\Delta y}\left(s_{r, k, 2} q_{r, 6}-s_{p, k, 2} q_{p, 6}\right),
\end{aligned}
$$$$
f_{x r, 2}=\rho \Delta y \Delta z \ddot{q}_{r, 2}-\kappa G \Delta y \Delta z \frac{\left(\frac{q_{r+1,2}-q_{r, 2}}{\Delta x}\right)-\left(\frac{q_{r, 2}-q_{r-1,2}}{\Delta x}\right)}{\Delta x}+
$$$$
+\frac{E \Delta z}{\Delta y}\left(q_{r, 2}-q_{p, 2}\right)-\frac{E \Delta z}{\Delta y}\left(s_{r, k, 3} q_{r, 4}-s_{p, k, 3} q_{p, 4}\right)+
$$$$
+\frac{\kappa G \Delta y \Delta z}{2}\left(\frac{q_{r+1,6}-q_{r, 6}}{\Delta x}\right)+\frac{\kappa G \Delta y \Delta z}{2}\left(\frac{q_{r, 6}-q_{r-1,6}}{\Delta x}\right),
$$$$
f_{x r, 3}=\rho \Delta y \Delta z \ddot{q}_{r, 3}-\kappa G \Delta y \Delta z \frac{\frac{\left(q_{r+1,3}-q_{r, 3}\right)}{\Delta x}-\frac{\left(q_{r, 3}-q_{r-1,3}\right)}{\Delta x}}{\Delta x}+
$$$$
+\frac{\kappa G \Delta z}{\Delta y}\left(q_{r, 3}-q_{p, 3}\right)+\frac{\kappa G \Delta z}{\Delta y}\left(s_{r, k, 2} q_{r, 4}-s_{p, k, 2} q_{p, 4}\right)+
$$$$
-\frac{\kappa G \Delta y \Delta z}{2}\left(\frac{q_{r+1,5}-q_{r, 5}}{\Delta x}\right)-\frac{\kappa G \Delta y \Delta z}{2}\left(\frac{q_{r, 5}-q_{r-1,5}}{\Delta x}\right),
$$$$
f_{x r, 4}=\rho I_{0 x} \ddot{q}_{r, 4}-G I_{0 x} \frac{\left(\frac{q_{r+1,4}-q_{r, 4}}{\Delta x}\right)-\left(\frac{q_{r, 4}-q_{r-1,4}}{\Delta x}\right)}{\Delta x}+
$$$$
+\frac{E I_{x y}}{\Delta y \Delta x}\left(q_{r, 4}-q_{p, 4}\right)+\frac{\kappa G \Delta z}{\Delta y}\left(s_{r, k, 2} q_{r, 3}-s_{r, k, 2} q_{p, 3}\right)+
$$$$
+\frac{\kappa G \Delta z}{\Delta y}\left(s_{r, k, 2}^{2} q_{r, 4}-s_{r, k, 2} s_{p, k, 2} q_{p, 4}\right)+
$$$$
+\frac{E \Delta z}{\Delta y}\left(s_{r, k, 3}^{2} q_{r, 4}-s_{r, k, 3} s_{p, k, 3} q_{p, 4}\right)+\frac{E \Delta z}{\Delta y}\left(s_{r, k, 3} q_{p, 2}-s_{r, k, 3} q_{r, 2}\right),
$$$$
f_{x r, 5}=\rho \frac{\Delta y}{\Delta x} \frac{1}{12} \Delta x \Delta z(\overbrace{\Delta x^{2}}^{=0}+\Delta z^{2}) \ddot{q}_{r, 5}+
$$$$
-E I_{y x} \frac{\left(\frac{q_{r+1,5}-q_{r, 5}}{\Delta x}\right)-\left(\frac{q_{r, 5}-q_{r-1,5}}{\Delta x}\right)}{\Delta x}+
$$

$$
+\frac{G \frac{1}{12} \Delta x \Delta z(\overbrace{\Delta x^{2}}^{=0}+\Delta z^{2})}{\Delta x \Delta y}\left(q_{r, 5}-q_{p, 5}\right)+
$$$$
+\frac{\kappa G \Delta y \Delta z}{\Delta x} \frac{\Delta x}{2}\left(\frac{q_{r, 3}-q_{r-1,3}}{\Delta x}\right)+\frac{\kappa G \Delta y \Delta z}{\Delta x} \frac{\Delta x}{2}\left(\frac{q_{r+1,3}-q_{r, 3}}{\Delta x}\right)+
$$$$
+\frac{\kappa G \Delta y \Delta z}{\Delta x \Delta x}\left(\frac{\Delta x}{2}\right)^{2}\left(q_{r, 5}+q_{r-1,5}\right)+\frac{\kappa G \Delta y \Delta z}{\Delta x \Delta x}\left(\frac{\Delta x}{2}\right)^{2}\left(q_{r, 5}+q_{r+1,5}\right)+
$$$$
+\frac{\kappa G \Delta x \Delta z}{\Delta y \Delta x} s_{r, k, 3}\left(q_{r, 1}-q_{p, 1}\right)+\frac{\kappa G \Delta x \Delta z}{\Delta y \Delta x}\left(s_{r, k, 3}^{2} q_{r, 5}-s_{r, k, 3} s_{p, k, 3} q_{p, 5}\right)+
$$$$
-\frac{\kappa G \Delta x \Delta z}{\Delta y \Delta x}\left(s_{r, k, 2} s_{r, k, 3} q_{r, 6}-s_{r, k, 3} s_{p, k, 2} q_{p, 6}\right),
$$

$$
\begin{aligned}
& f_{x r, 6}=\rho \frac{\Delta z}{\Delta x} \frac{1}{12} \Delta x \Delta y(\Delta y^{2}+\overbrace{\Delta x^{2}}^{=0} \ddot{q}_{r, 6}+ \\
& -E I_{z x} \frac{\left(\frac{q_{r+1,6}-q_{r, 6}}{\Delta x}\right)-\left(\frac{q_{r, 6}-q_{r-1,6}}{\Delta x}\right)}{\Delta x}+ \\
& +\frac{\kappa G \Delta y \Delta z}{\Delta x} \frac{\Delta x}{2}\left(\frac{q_{r, 2}-q_{r+1,2}}{\Delta x}\right)+\frac{\kappa G \Delta y \Delta z}{\Delta x} \frac{\Delta x}{2}\left(\frac{q_{r-1,2}-q_{r, 2}}{\Delta x}\right)+ \\
& +\frac{E \frac{1}{12} \Delta z \Delta x \Delta x^{2}}{\Delta y \Delta x}\left(q_{r, 6}-q_{p, 6}\right)- \\
& -\frac{\kappa G \Delta x \Delta z}{\Delta y \Delta x} S_{r, k, 2}\left(q_{r, 1}-q_{p, 1}\right)+ \\
& -\frac{\kappa G \Delta x \Delta z}{\Delta y \Delta x}\left(s_{r, k, 2} S_{r, k, 3} q_{r, 5}-s_{r, k, 2} s_{p, k, 3} q_{p, 5}\right)+ \\
& +\frac{\kappa G \Delta y \Delta z}{\Delta x \Delta x}\left(\frac{\Delta x}{2}\right)^{2}\left(q_{r, 6}+q_{r-1,6}\right)+ \\
& +\frac{\kappa G \Delta y \Delta z}{\Delta x \Delta x}\left(\frac{\Delta x}{2}\right)^{2}\left(q_{r, 6}+q_{r+1,6}\right)+ \\
& +\frac{\kappa G \Delta x \Delta z}{\Delta y \Delta x}\left(s_{r, k, 2}^{2} q_{r, 6}-s_{r, k, 2} s_{p, k, 2} q_{p, 6}\right),
\end{aligned}
$$

where $f_{x r, \alpha}$ is the distributed force along the $x_{1}$-axis applied to the $r$-th element (excitation) in the $x_{\alpha}$-axis direction (Fig. 2).

By assuming $\Delta x \rightarrow 0$, we can obtain the following partial differential equations:

$$
\begin{aligned}
& f_{x r, 1}=\rho A \ddot{q}_{r, 1}-E A q_{r, 1}^{\prime \prime}+ \\
& +\frac{\kappa G \Delta z}{\Delta y}\left(q_{r, 1}-q_{p, 1}\right)+\frac{\kappa G \Delta z}{\Delta y}\left(s_{r, k, 3} q_{r, 5}-s_{p, k, 3} q_{p, 5}\right)+ \\
& -\frac{\kappa G \Delta z}{\Delta y}\left(s_{r, k, 2} q_{r, 6}-s_{p, k, 2} q_{p, 6}\right), \\
& f_{x r, 2}=\rho A \ddot{q}_{r, 2}-\kappa G A q_{r, 2}^{\prime \prime}+\kappa G A q_{r, 6}^{\prime}+ \\
& +\frac{E \Delta z}{\Delta y}\left(q_{r, 2}-q_{p, 2}\right)-\frac{E \Delta z}{\Delta y}\left(s_{r, k, 3} q_{r, 4}-s_{p, k, 3} q_{p, 4}\right), \\
& f_{x r, 3}=\rho A \ddot{q}_{r, 3}-\kappa G A q_{r, 3}^{\prime \prime}-\kappa G A q_{r, 5}^{\prime} \\
& \frac{\kappa G \Delta z}{\Delta y}\left(q_{r, 3}-q_{p, 3}\right)+\frac{\kappa G \Delta z}{\Delta y}\left(s_{r, k, 2} q_{r, 4}-s_{p, k, 2} q_{p, 4}\right), \\
& f_{x r, 4}=\rho I_{0 x} \ddot{q}_{r, 4}-G I_{0 x} q_{r, 4}^{\prime \prime}+ \\
& +\frac{E \Delta z}{12 \Delta y}\left(q_{r, 4}-q_{p, 4}\right)+\frac{\kappa G \Delta z}{\Delta y}\left(s_{r, k, 2} q_{r, 3}-s_{r, k, 2} q_{p, 3}\right)+ \\
& +\frac{\kappa G \Delta z}{\Delta y}\left(s_{r, k, 2}^{2} q_{r, 4}-s_{r, k, 2} s_{p, k, 2} q_{p, 4}\right)+ \\
& +\frac{E \Delta z}{\Delta y}\left(s_{r, k, 3}^{2} q_{r, 4}-s_{r, k, 3} S_{p, k, 3} q_{p, 4}\right)+ \\
& +\frac{E \Delta z}{\Delta y}\left(s_{r, k, 3} q_{p, 2}-s_{r, k, 3} q_{r, 2}\right), \\
&
\end{aligned}
$$


$f_{x r, 5}=\rho I_{y x} \ddot{q}_{r, 5}-E I_{y x} q_{r, 5}^{\prime \prime}+\kappa G A q_{r, 3}^{\prime}+\kappa G A q_{r, 5}$

$\frac{G \Delta z^{3}}{12 \Delta y}\left(q_{r, 5}-q_{p, 5}\right)+\frac{\kappa G \Delta z}{\Delta y} S_{r, k, 3}\left(q_{r, 1}-q_{p, 1}\right)+$

$+\frac{\kappa G \Delta z}{\Delta y}\left(s_{r, k, 3}^{2} q_{r, 5}-s_{r, k, 3} s_{p, k, 3} q_{p, 5}\right)+$

$-\frac{\kappa G \Delta z}{\Delta y}\left(s_{r, k, 2} s_{r, k, 3} q_{r, 6}-s_{r, k, 3} s_{p, k, 2} q_{p, 6}\right)$,

$f_{x r, 6}=\rho I_{z x} \ddot{q}_{r, 6}-E I_{z x} q_{r, 6}^{\prime \prime}-\kappa G A q_{r, 2}^{\prime}+\kappa G A q_{r, 6}+$

$-\frac{\kappa G \Delta z}{\Delta y} s_{r, k, 2}\left(q_{r, 1}-q_{p, 1}\right)+\frac{\kappa G \Delta z}{\Delta y}\left(s_{r, k, 2}^{2} q_{r, 6}-s_{r, k, 2} s_{p, k, 2} q_{p, 6}\right)+$

$-\frac{\kappa G \Delta z}{\Delta y}\left(s_{r, k, 2} s_{r, k, 3} q_{r, 5}-s_{r, k, 2} s_{p, k, 3} q_{p, 5}\right)$,

where:

$A=\Delta y \Delta z, I_{y x}=\frac{1}{12} \Delta y \Delta z^{3}, I_{z x}=\frac{1}{12} \Delta z \Delta y^{3}, \dot{q}=d / d t q, q^{\prime}=d / d x q$.

In order to obtain the general form of Equations $(19) \div(24)$, the following substitutions are applied:

$c_{x k, 1}=\frac{\kappa G \Delta z}{\Delta y}, c_{x k, 2}=\frac{E \Delta z}{\Delta y}, c_{x k, 3}=\frac{\kappa G \Delta z}{\Delta y}, c_{x k, 6}=\lim _{\Delta x \rightarrow 0} \frac{E I_{z y}}{\Delta y \Delta x}=0$,

$c_{x k, 4}=\lim _{\Delta x \rightarrow 0} \frac{E I_{x y}}{\Delta y \Delta x}=\frac{E \Delta z^{3}}{12 \Delta y}, c_{x k, 5}=\lim _{\Delta x \rightarrow 0} \frac{G I_{0 y}}{\Delta y \Delta x}=\frac{G \Delta z^{3}}{12 \Delta y}$.

Thus Equations $(19) \div(24)$ can be presented in the final form:

$$
\begin{aligned}
& f_{x r, 1}=\rho A \ddot{q}_{r, 1}-E A q_{r, 1}^{\prime \prime}+ \\
& +c_{x k, 1}\left(q_{r, 1}-q_{p, 1}\right)+c_{x k, 1}\left(s_{r, k, 3} q_{i, j, 5}-s_{p, k, 3} q_{p, 5}\right)+ \\
& -c_{x k, 1}\left(s_{r, k, 2} q_{r, 6}-s_{p, k, 2} q_{p, 6}\right) \text {, } \\
& f_{x r, 2}=\rho A \ddot{q}_{r, 2}-\kappa G A q_{r, 2}^{\prime \prime}+\kappa G A q_{r, 6}^{\prime}+ \\
& +c_{x k, 2}\left(q_{r, 2}-q_{p, 2}\right)-c_{x k, 2}\left(s_{r, k, 3} q_{r, 4}-s_{p, k, 3} q_{p, 4}\right) \text {. } \\
& f_{x r, 4}=\rho I_{0 x} \ddot{q}_{r, 4}-G I_{0 x} q_{r, 4}^{\prime \prime}+ \\
& +c_{x k, 4}\left(q_{r, 4}-q_{p, 4}\right)+c_{x k, 3}\left(s_{r, k, 2} q_{r, 3}-s_{r, k, 2} q_{p, 3}\right)+ \\
& +c_{x k, 3}\left(s_{r, k, 2}^{2} q_{r, 4}-s_{r, k, 2} s_{p, k, 2} q_{p, 4}\right)+ \\
& +c_{x k, 2}\left(s_{r, k, 3}^{2} q_{r, 4}-s_{r, k, 3} s_{p, k, 3} q_{p, 4}\right)+c_{x k, 2}\left(s_{r, k, 3} q_{p, 2}-s_{r, k, 3} q_{r, 2}\right) \text {, } \\
& f_{x r, 4}=\rho I_{0 x} \ddot{q}_{r, 4}-G I_{0 x} q_{r, 4}^{\prime \prime}+ \\
& +c_{x k, 4}\left(q_{r, 4}-q_{p, 4}\right)+c_{x k, 3}\left(s_{r, k, 2} q_{r, 3}-s_{r, k, 2} q_{p, 3}\right)+ \\
& +c_{x k, 3}\left(s_{r, k, 2}^{2} q_{r, 4}-s_{r, k, 2} s_{p, k, 2} q_{p, 4}\right)+ \\
& +c_{x k, 2}\left(s_{r, k, 3}^{2} q_{r, 4}-s_{r, k, 3} s_{p, k, 3} q_{p, 4}\right)+c_{x k, 2}\left(s_{r, k, 3} q_{p, 2}-s_{r, k, 3} q_{r, 2}\right) \text {, } \\
& f_{x r, 5}=\rho I_{y x} \ddot{q}_{r, 5}-E I_{y x} q_{r, 5}^{\prime \prime}+\kappa G A q_{r, 3}^{\prime}+\kappa G A q_{r, 5}+ \\
& +c_{x k, 5}\left(q_{r, 5}-q_{p, 5}\right)+c_{x k, 1} s_{r, k, 3}\left(q_{r, 1}-q_{p, 1}\right)+ \\
& +c_{x k, 1}\left(s_{r, k, 3}^{2} q_{r, 5}-s_{r, k, 3} s_{p, k, 3} q_{p, 5}\right)+ \\
& -c_{x k, 1}\left(s_{r, k, 2} s_{r, k, 3} q_{r, 6}-s_{r, k, 3} s_{p, k, 2} q_{p, 6}\right) \text {, } \\
& f_{x r, 6}=\rho I_{z x} \ddot{q}_{r, 6}-E I_{z x} q_{r, 6}^{\prime \prime}-\kappa G A q_{r, 2}^{\prime}+\kappa G A q_{r, 6}+ \\
& -c_{x k, 1} s_{r, k, 2}\left(q_{r, 1}-q_{p, 1}\right)-c_{x k, 1}\left(s_{r, k, 2} s_{r, k, 3} q_{r, 5}-s_{r, k, 2} s_{p, k, 3} q_{p, 5}\right)+ \\
& +c_{x k, 1}\left(s_{r, k, 2}^{2} q_{r, 6}-s_{r, k, 2} s_{p, k, 2} q_{p, 6}\right) \text {. }
\end{aligned}
$$

The above equations are obtained for prism $r$ (connected to prism $p$ ). However, by exchanging subscripts $r \leftrightarrow p$, we can easily obtain appropriate equations related to prism $p$.

The general equations for the whole system, consisting in this particular case of $r$ and $p$ elements, can be written in the matrix form

$$
\boldsymbol{A}_{02} \ddot{\boldsymbol{q}}(x, t)+\boldsymbol{A}_{20} \boldsymbol{q}^{\prime \prime}(x, t)+\boldsymbol{A}_{10} \boldsymbol{q}^{\prime}(x, t)+\boldsymbol{A}_{00} \boldsymbol{q}(x, t)=\boldsymbol{f}(x, t)
$$

with the boundary conditions

$\boldsymbol{M}_{j} \boldsymbol{q}(0, t)+\boldsymbol{N}_{j} \boldsymbol{q}(l, t)=\boldsymbol{\gamma}_{j}(t), \quad j=1,2$

where:

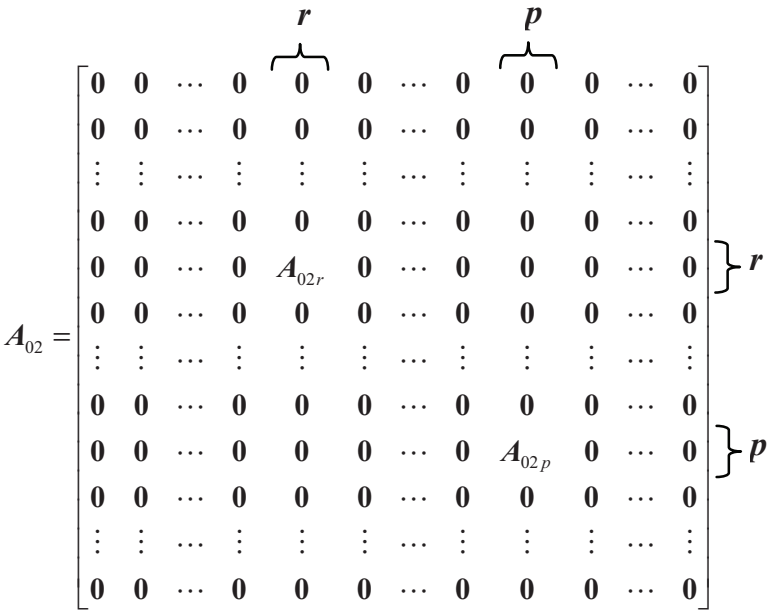

or $\boldsymbol{A}_{02}=\operatorname{diag}\left[\mathbf{0 , 0}, \cdots, \mathbf{0}, \boldsymbol{A}_{02 r}, \mathbf{0}, \cdots, \mathbf{0}, \boldsymbol{A}_{02 p}, \mathbf{0}, \cdots, \mathbf{0}\right]$,

where $\boldsymbol{A}_{02 r}=\operatorname{diag}\left[\rho A, \rho A, \rho A, \rho I_{0 x}, \rho I_{y x}, \rho I_{z x}\right]$,

$\boldsymbol{A}_{02 p}$ can be obtained from the matrix $\boldsymbol{A}_{02 r}$ by replacing the parameters and dimensions of the $r$ prism with appropriate parameters of the $p$ prism,

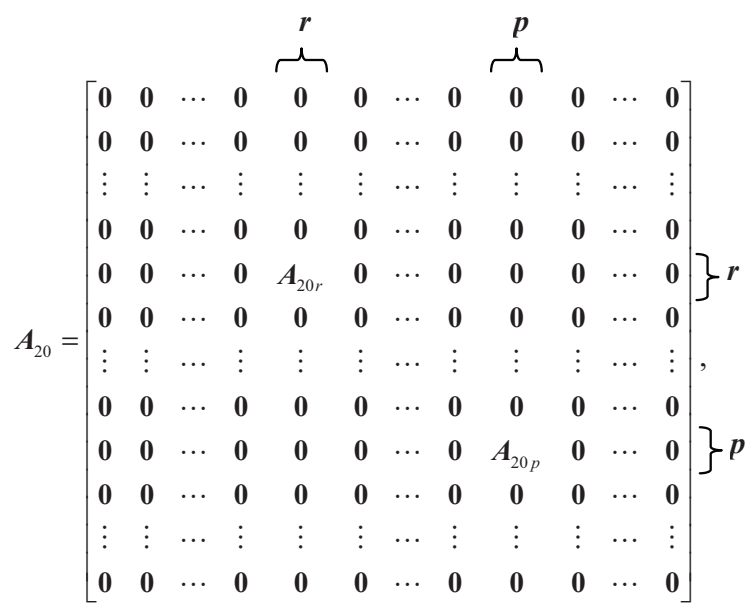

or $\boldsymbol{A}_{20}=\operatorname{diag}\left[\mathbf{0}, \mathbf{0}, \cdots, \mathbf{0}, \boldsymbol{A}_{20 r}, \mathbf{0}, \cdots, \mathbf{0}, \boldsymbol{A}_{20 p}, \mathbf{0}, \cdots, \mathbf{0}\right]$,

where $\boldsymbol{A}_{20 r}=\operatorname{diag}\left[-E A,-\kappa G A,-\kappa G A,-G I_{0 x},-E I_{y x},-E I_{z x}\right]$. 
$\boldsymbol{A}_{20 p}$ can be obtained from matrix $\boldsymbol{A}_{20 r}$ by replacing the parameters and dimensions of the $r$ prism with appropriate parameters of the $p$ prism,

$$
\left.\boldsymbol{A}_{10}=\left[\begin{array}{cccccccccccc}
\mathbf{0} & \mathbf{0} & \cdots & \mathbf{0} & \mathbf{0} & \mathbf{0} & \cdots & \mathbf{0} & \mathbf{0} & \mathbf{0} & \cdots & \mathbf{0} \\
\mathbf{0} & \mathbf{0} & \cdots & \mathbf{0} & \mathbf{0} & \mathbf{0} & \cdots & \mathbf{0} & \mathbf{0} & \mathbf{0} & \cdots & \mathbf{0} \\
\vdots & \vdots & \cdots & \vdots & \vdots & \vdots & \cdots & \vdots & \vdots & \vdots & \cdots & \vdots \\
\mathbf{0} & \mathbf{0} & \cdots & \mathbf{0} & \mathbf{0} & \mathbf{0} & \cdots & \mathbf{0} & \mathbf{0} & \mathbf{0} & \cdots & \mathbf{0} \\
\mathbf{0} & \mathbf{0} & \cdots & \mathbf{0} & \boldsymbol{A}_{10 r} & \mathbf{0} & \cdots & \mathbf{0} & \mathbf{0} & \mathbf{0} & \cdots & \mathbf{0} \\
\mathbf{0} & \mathbf{0} & \cdots & \mathbf{0} & \mathbf{0} & \mathbf{0} & \cdots & \mathbf{0} & \mathbf{0} & \mathbf{0} & \cdots & \mathbf{0} \\
\vdots & \vdots & \cdots & \vdots & \vdots & \vdots & \cdots & \vdots & \vdots & \vdots & \cdots & \vdots \\
\mathbf{0} & \mathbf{0} & \cdots & \mathbf{0} & \mathbf{0} & \mathbf{0} & \cdots & \mathbf{0} & \mathbf{0} & \mathbf{0} & \cdots & \mathbf{0} \\
\mathbf{0} & \mathbf{0} & \cdots & \mathbf{0} & \mathbf{0} & \mathbf{0} & \cdots & \mathbf{0} & \boldsymbol{A}_{10 p} & \mathbf{0} & \cdots & \mathbf{0} \\
\mathbf{0} & \mathbf{0} & \cdots & \mathbf{0} & \mathbf{0} & \mathbf{0} & \cdots & \mathbf{0} & \mathbf{0} & \mathbf{0} & \cdots & \mathbf{0} \\
\vdots & \vdots & \cdots & \vdots & \vdots & \vdots & \cdots & \vdots & \vdots & \vdots & \cdots & \vdots \\
\mathbf{0} & \mathbf{0} & \cdots & \mathbf{0} & \mathbf{0} & \mathbf{0} & \cdots & \mathbf{0} & \mathbf{0} & \mathbf{0} & \cdots & \mathbf{0}
\end{array}\right]\right\} \boldsymbol{p}
$$

or $\boldsymbol{A}_{10}=\operatorname{diag}\left[\mathbf{0}, \mathbf{0}, \cdots, \mathbf{0}, \boldsymbol{A}_{10 r}, \mathbf{0}, \cdots, \mathbf{0}, \boldsymbol{A}_{10 p}, \mathbf{0}, \cdots, \mathbf{0}\right]$, where

$$
\boldsymbol{A}_{10 r}=\left[\begin{array}{cccccc}
0 & 0 & 0 & 0 & 0 & 0 \\
0 & 0 & 0 & 0 & 0 & \kappa G A \\
0 & 0 & 0 & 0 & -\kappa G A & 0 \\
0 & 0 & 0 & 0 & 0 & 0 \\
0 & 0 & \kappa G A & 0 & 0 & 0 \\
0 & -\kappa G A & 0 & 0 & 0 & 0
\end{array}\right],
$$

$\boldsymbol{A}_{10 p}$ can be obtained from the matrix $\boldsymbol{A}_{10 r}$ by replacing the parameters and dimensions of the $r$ prism with the appropriate parameters and dimensions of the $p$ prism,

$\boldsymbol{A}_{00}=\boldsymbol{A}_{k}+\boldsymbol{A}_{s}$,

where $\boldsymbol{A}_{k}$ has a form analogous to the stiffness matrix $\widetilde{\boldsymbol{K}}_{k}[5]$ and

$$
A_{r r k}=\left[\begin{array}{cccccc}
a_{r r k 11} & 0 & 0 & 0 & a_{r r k 15} & a_{r r k 16} \\
& a_{r r k 22} & 0 & a_{r r k 24} & 0 & 0 \\
& & a_{r r k 33} & a_{r r k 34} & 0 & 0 \\
& & & a_{r r k 44} & 0 & 0 \\
& & & & a_{r r k 55} & a_{r r k 56} \\
s y m & & & & & a_{r r k 66}
\end{array}\right],
$$

$a_{r r k 11}=c_{x k 1}, a_{r r k 15}=c_{x k 1} s_{r k 3}, a_{r r k 16}=-c_{x k 1} s_{r k 2}$,

$a_{r r k 22}=c_{x k 2}, a_{r r k 24}=-c_{x k 2} s_{r k 3}$,

$a_{r r k 33}=c_{x k 3}, a_{r r k 34}=c_{x k 3} s_{r k 2}$,

$a_{r r k 44}=c_{x k 4}+c_{x k 2} s_{r k 3}^{2}+c_{x k 3} s_{r k 2}^{2}$,

$a_{r r k 55}=c_{x k 5}+c_{x k 1} s_{r k 3}^{2}, a_{r r k 56}=-c_{x k 1} s_{r k 2} s_{r k 3}$,

$a_{r r k 66}=c_{x k 6}+c_{x k 1} s_{r k 2}^{2}$,

$\boldsymbol{A}_{r p k}=\left[\begin{array}{cccccc}a_{r p k 11} & 0 & 0 & 0 & a_{r p k 15} & a_{r p k 16} \\ 0 & a_{r p k 22} & 0 & a_{r p k 24} & 0 & 0 \\ 0 & 0 & a_{r p k 33} & a_{r p k 34} & 0 & 0 \\ 0 & a_{r p k 42} & a_{r p k 43} & a_{r p k 44} & 0 & 0 \\ a_{r p k 51} & 0 & 0 & 0 & a_{r p k 55} & a_{r p k 56} \\ a_{r p k 61} & 0 & 0 & 0 & a_{r p k 65} & a_{r p k 66}\end{array}\right]$

$a_{r p k 11}=-c_{x k 1}, a_{r p k 15}=-c_{x k 1} s_{p k 3}, a_{r p k 16}=c_{x k 1} s_{p k 2}$,

$a_{r p k 22}=-c_{x k 2}, a_{r p k 24}=c_{x k 2} s_{p k 3}$,

$a_{r p k 33}=-c_{x k 3}, a_{r p k 34}=-c_{x k 3} s_{p k 2}$,

$a_{r p k 42}=c_{x k 2} s_{r k 3}, a_{r p k 43}=-c_{x k 3} s_{r k 2}, a_{r p k 44}=-c_{x k 4}-c_{x k 2} s_{r k 3} s_{p k 3}-c_{x k 3} s_{r k 2} s_{p k 2}$,

$a_{r p k 51}=-c_{x k 1} s_{r k 3}, a_{r p k 55}=-c_{x k 5}-c_{x k 1} s_{r k 3} s_{p k 3}, a_{r p k 56}=c_{x k 1} s_{r k 3} s_{p k 2}$,

$a_{r p k 61}=c_{x k 1} s_{r k 2}, a_{r p k 65}=c_{x k 1} s_{r k 2} s_{p k 3}, a_{r p k 66}=-c_{x k 6}-c_{x k 1} s_{r k 2} s_{p k 2}$,

$\boldsymbol{A}_{p r k}=\boldsymbol{A}_{r p k}^{T}$,

$\boldsymbol{A}_{p p k}$ is obtained from the matrix $\boldsymbol{A}_{r r k}$ by replacing indexes $r$ with $p$,

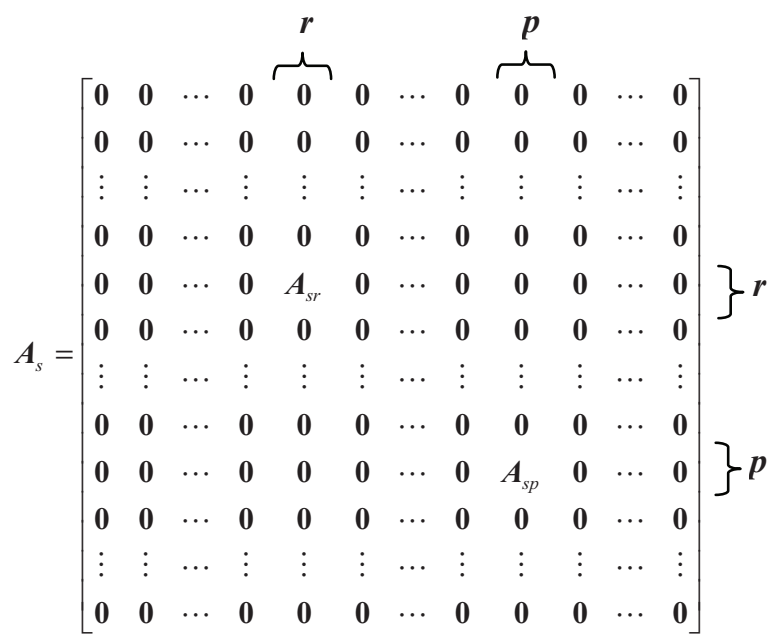


and

$\boldsymbol{A}_{s r}=\left[\begin{array}{cccccc}0 & 0 & 0 & 0 & 0 & 0 \\ 0 & 0 & 0 & 0 & 0 & 0 \\ 0 & 0 & 0 & 0 & 0 & 0 \\ 0 & 0 & 0 & 0 & 0 & 0 \\ 0 & 0 & 0 & 0 & \kappa G A & 0 \\ 0 & 0 & 0 & 0 & 0 & \kappa G A\end{array}\right]$.

$\boldsymbol{A}_{s p}$ is obtained from the matrix $\boldsymbol{A}_{s r}$ by replacing the parameters and dimensions of the $r$ prism with the appropriate parameters and dimensions of the $p$ prism.

The equations derived in the above way were then verified in three steps. In the first step, continuous parts of Equations (26) $\div(31)$ were compared to the appropriate components of the well-known equations describing one-dimensional elements with distributed parameters. As can be seen, the continuous part (first line) of Equation (26) is the same as the equation for a longitudinal bar element. In turn, the continuous parts of Equations (27) and (31) are coupled together and are the same as the Timoshenko beam model equations (vibrating in the $x_{1}-x_{2}$ plane). Similarly, Equations (28) and (30) also describe the Timoshenko beam model, but vibrating in the perpendicular plane $x_{1}-x_{3}$. Lastly, the continuous part of Equation (29) expresses a torsion bar element.

In the second step, Equations (26) $\div(31)$ were written in the matrix form and the obtained stiffness matrix $\boldsymbol{A}_{k}$ (38) was compared to the corresponding matrix $\widetilde{\boldsymbol{K}}_{k}$ which describes the discrete model in the rigid finite element method $[5,13$, 14]. As it turns out, the discrete parts of Equations (26) $\div(31)$ are analogous to those in the rigid finite element method [5]. In the last step, easy examples were used and presented in this paper, some of which have analytical solutions and are thus easily verifiable.

A global model for the whole system is built in the same way as the finite element method (FEM) model. Global matrices $\boldsymbol{A}_{02}, \boldsymbol{A}_{20}, \boldsymbol{A}_{10}$ include sub-matrices of each prism element, located on a main diagonal. Matrix $\boldsymbol{A}_{00}$ is the sum of all the prism element stiffness matrices in the global system.

To solve the partial differential equation (32), the distributed transfer function method $[15,16,18]$ is used. This method gives semi-analytical results for the tree-dimensional structure. To use it, Equation (32) is to be subjected to Laplace transformation, after which it takes the form

$\left(\boldsymbol{A}_{02} s^{2}+\boldsymbol{A}_{20} \frac{\partial}{\partial x^{2}}+\boldsymbol{A}_{10} \frac{\partial}{\partial x}+\boldsymbol{A}_{00}\right) \overline{\boldsymbol{q}}(x, s)=\overline{\boldsymbol{f}}(x, s)$

with the boundary conditions

$\overline{\boldsymbol{M}}_{j} \overline{\boldsymbol{q}}(0, s)+\overline{\boldsymbol{N}}_{j} \overline{\boldsymbol{q}}(l, s)=\overline{\boldsymbol{\gamma}}_{j}(s), j=1,2$,

where:

$\overline{\boldsymbol{q}}(\bullet, s), \overline{\boldsymbol{f}}(\bullet, s)$ denote the Laplace transformation of the corresponding functions $q(\bullet, t), f(\bullet, t)$, respectively, and $\overline{\boldsymbol{M}}_{j}, \overline{\boldsymbol{N}}_{j}$ are the operator matrices formed from the matrices $\boldsymbol{M}_{j}, \boldsymbol{N}_{j}$ by replacing the time-derivative operators $\partial / \partial t$ and $\partial^{2} / \partial t^{2}$ with $s$ and $s^{2}$, respectively.

Equation (40) can be transformed into the set of first-order equations (state equations):

$\frac{\partial}{\partial x} \boldsymbol{\eta}(x, s)=\boldsymbol{F}(s) \boldsymbol{\eta}(x, s)+\boldsymbol{u}(x, s)$

$\boldsymbol{M}(s) \boldsymbol{\eta}(0, s)+\boldsymbol{N}(s) \boldsymbol{\eta}(l, s)=\boldsymbol{\gamma}(s)$

where:

$$
\begin{aligned}
& \boldsymbol{\eta}(x, s)=\left[\begin{array}{l}
\boldsymbol{\eta}_{1}(x, s) \\
\boldsymbol{\eta}_{2}(x, s)
\end{array}\right]=\left[\begin{array}{c}
\overline{\boldsymbol{q}}(x, s) \\
\frac{\partial \overline{\boldsymbol{q}}(x, s)}{\partial x}
\end{array}\right], \\
& \boldsymbol{F}=\left[\begin{array}{c:c}
\mathbf{0} & \boldsymbol{I} \\
\hdashline-\boldsymbol{A}_{20}^{-1}\left(\boldsymbol{A}_{02} s^{2}+\boldsymbol{A}_{00}\right) & -\boldsymbol{A}_{20}^{-1} \boldsymbol{A}_{10}
\end{array}\right], \\
& \boldsymbol{u}(x, s)=\left[\begin{array}{l}
\boldsymbol{u}_{1}(x, s) \\
\boldsymbol{u}_{2}(x, s)
\end{array}\right]=\left[\begin{array}{c}
\mathbf{0} \\
-\boldsymbol{A}_{20}^{-1} \overline{\boldsymbol{f}}(x, s)
\end{array}\right], \boldsymbol{M}=\left[\begin{array}{cc}
\boldsymbol{M}_{1} & \boldsymbol{M}_{2} \\
\mathbf{0} & \mathbf{0}
\end{array}\right] \text {, } \\
& \boldsymbol{N}=\left[\begin{array}{cc}
\mathbf{0} & \mathbf{0} \\
\boldsymbol{N}_{1} & \boldsymbol{N}_{2}
\end{array}\right], \boldsymbol{\gamma}(s)=\left[\begin{array}{l}
\boldsymbol{\gamma}_{1}(s) \\
\gamma_{2}(s)
\end{array}\right] \text {. }
\end{aligned}
$$

The solution to Equation (42) can be presented as $[12,18]$

$$
\boldsymbol{\eta}(x, s)=\int_{x_{1}}^{x_{2}} \boldsymbol{G}(x, \xi, s) \boldsymbol{u}(\xi, s) d \xi+\boldsymbol{H}(x, s) \boldsymbol{\gamma}(s)
$$

where:

$$
\begin{aligned}
& \boldsymbol{G}(x, \xi, s)= \\
& =\left\{\begin{array}{cc}
e^{F(s) x}\left[\boldsymbol{M}(s) e^{F(s) x_{1}}+\boldsymbol{N}(s) e^{F(s) x_{2}}\right]^{-1} \boldsymbol{M}(s) e^{F(s)\left(x_{1}-\xi\right)}, & \xi \leq x \\
-e^{F(s) x}\left[\boldsymbol{M}(s) e^{F(s) x_{1}}+\boldsymbol{N}(s) e^{F(s) x_{2}}\right]^{-1} \boldsymbol{N}(s) e^{F(s)\left(x_{2}-\xi\right)}, & \xi \geq x
\end{array}\right. \\
& \boldsymbol{H}(x, s)=e^{\boldsymbol{F}(s) x}\left[\boldsymbol{M}(s) e^{\boldsymbol{F}(s) x_{1}}+\boldsymbol{N}(s) e^{\boldsymbol{F}(s) x_{2}}\right]^{-1}
\end{aligned}
$$

If we denote:

$$
\begin{aligned}
& \boldsymbol{G}(x, \xi, s)=\left[\begin{array}{ll}
\boldsymbol{G}_{11}(x, \xi, s) & \boldsymbol{G}_{12}(x, \xi, s) \\
\boldsymbol{G}_{21}(x, \xi, s) & \boldsymbol{G}_{22}(x, \xi, s)
\end{array}\right], \\
& \boldsymbol{H}(x, s)=\left[\begin{array}{ll}
\boldsymbol{H}_{11}(x, s) & \boldsymbol{H}_{12}(x, s) \\
\boldsymbol{H}_{21}(x, s) & \boldsymbol{H}_{22}(x, s)
\end{array}\right],
\end{aligned}
$$

the solution to Eq. (44) can be written as

$$
\boldsymbol{\eta}_{i}(x, s)=\sum_{j=1}^{2} \int_{x_{1}}^{x_{2}} \boldsymbol{G}_{i j}(x, \xi, s) \boldsymbol{u}_{j}(\xi, s) d \xi+\sum_{j=1}^{2} \boldsymbol{H}_{i j}(x, s) \boldsymbol{\gamma}_{j}(s),
$$

$i=1,2$, or

$\boldsymbol{\eta}_{i}(x, s)=\int_{x_{1}}^{x_{2}} \boldsymbol{G}_{i 2}(x, \xi, s) \boldsymbol{A}_{20}^{-1} \overline{\boldsymbol{f}}(\xi, s) d \xi+\sum_{j=1}^{2} \boldsymbol{H}_{i j}(x, s) \boldsymbol{\gamma}_{j}(s), i=1,2$ 
where the expression

$$
\boldsymbol{K}(x, \xi, s)=\left[\begin{array}{l}
\boldsymbol{G}_{12}(x, \xi, s) \boldsymbol{A}_{20}^{-1} \\
\boldsymbol{G}_{22}(x, \xi, s) \boldsymbol{A}_{20}^{-1}
\end{array}\right]
$$

is the matrix of transfer function between the $i$-th output (row) and the $j$-th input (column). In the case of a force concentrated at point $x_{0}$, the relation (50) can be written as

$\boldsymbol{\eta}_{i}(x, s)=\int_{0}^{1} \boldsymbol{G}_{i 2}(x, \xi, s) \boldsymbol{A}_{20}^{-1} \overline{\boldsymbol{f}}(\xi, s) \delta\left(\xi-x_{0}\right) d \xi+\sum_{j=1}^{2} \boldsymbol{H}_{i j}(x, s) \boldsymbol{\gamma}_{j}(s), i=1,2$

and after integrating

$\boldsymbol{\eta}_{i}(x, s)=\boldsymbol{G}_{i 2}\left(x, x_{0}, s\right) \boldsymbol{A}_{20}^{-1} \overline{\boldsymbol{f}}\left(x_{0}, s\right)+\sum_{j=1}^{2} \boldsymbol{H}_{i j}(x, s) \boldsymbol{\gamma}_{j}(s), \quad i=1,2$.

Assuming that the boundary conditions $\gamma(s)=\mathbf{0}$, from Eq. (53) we obtain the equation

$\boldsymbol{\eta}_{i}(x, s)=\boldsymbol{G}_{i 2}\left(x, x_{0}, s\right) \boldsymbol{A}_{20}^{-1} \overline{\boldsymbol{f}}\left(x_{0}, s\right)$.

The condition $\gamma(s)=\mathbf{0}$ occurs in most cases. It means that the external force does not act on beam supports [12].

In the specific case, when the excitation point $\xi=0.1$ and the response observation point $x=0.4$ and $\gamma(s)=\mathbf{0}$, the transfer function of the distributed parameter system is calculated by putting corresponding submatrices of the relation (45) into Eq. (51). In this way we obtain:

a) for $\xi<x$

$$
\boldsymbol{K}_{\xi<x}(s)=e^{F(s) \cdot 0.4}\left[\boldsymbol{M}(s) e^{F(s) \cdot 0}+\boldsymbol{N}(s) e^{F(s) \cdot 1}\right]^{-1} \boldsymbol{M}(s) e^{F(s) \cdot 0.1} \boldsymbol{A}_{20}^{-1}
$$

b) for $\xi>x$

$\boldsymbol{K}_{\xi<x}(s)=-e^{\boldsymbol{F}(s) \cdot 0.4}\left[\boldsymbol{M}(s) e^{F(s) \cdot 0}+\boldsymbol{N}(s) e^{\boldsymbol{F}(s) \cdot 1}\right]^{-1} \boldsymbol{M}(s) e^{F(s) \cdot 0.9} \boldsymbol{A}_{20}^{-1}$

After putting $s=j \omega$ into the transfer functions (55) and (56), the frequency characteristics can be calculated and the eigenvalues of the system may be determined.

\section{FIRST EXAMPLE OF METHOD APPLICATION}

As the first example, let us consider a simple beam fixed at both ends (Fig. 3 ) with the following data: $\mathrm{E}=2 \cdot 10^{11} \mathrm{~Pa}$, $\mathrm{G}=8 \cdot 10^{11} \mathrm{~Pa}, \rho=8000 \mathrm{~kg} / \mathrm{m}^{3}, \Delta y=0.25 \mathrm{~m}, \Delta z=0.25 \mathrm{~m}, l=1 \mathrm{~m}$, $\nu=0.25, \kappa=1$.

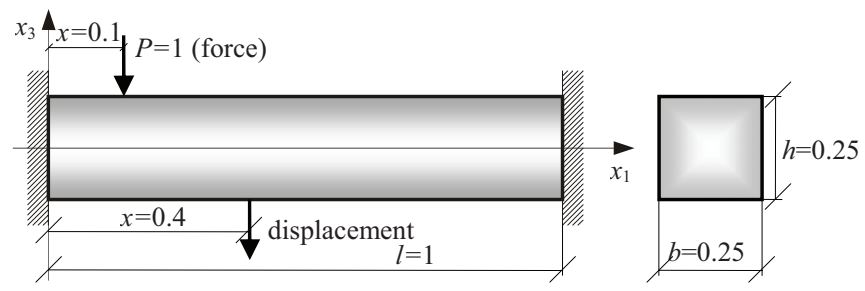

Fig. 3. Fixed beam
The calculations were performed for two cases. In the first case the beam was treated as a single prism, description of which comes down to the coupled two equations of the Timoshenko beam model, Eqs. (27) and (31), or (28) and (30) without discrete parts. In the second case the beam is divided into four prisms (Fig. 4) and four distributed spring elements. Each prism has three degrees of freedom: displacements along the $x_{1}$ and $x_{2}$-axes, and angular displacement around the $x_{3}$-axis.

The stiffness coefficients of the distributed spring layers are calculated using the formulas:

$c_{x 11}=c_{x 13}=c_{x 41}=c_{x 43}=\frac{\kappa G \Delta z}{2 \Delta y}, c_{x 15}=c_{x 45}=\frac{G \Delta z^{3}}{2 \cdot 12 \cdot \Delta y}$,

$c_{x 21}=c_{x 31}=c_{x 22}=c_{x 32}=\frac{\kappa G \Delta y}{2 \Delta z}, c_{x 25}=c_{x 35}=0, c_{x 23}=c_{x 33}=\frac{E \Delta y}{2 \Delta z}$

and the fixing coordinates:

$$
\begin{aligned}
& s_{1,1,2}=s_{3,4,2}=s_{1,2,2}=s_{3,2,2}=\frac{\Delta y}{4}, \\
& s_{2,1,2}=s_{4,4,2}=s_{2,3,2}=s_{4,3,2}=-\frac{\Delta y}{4}, \\
& s_{1,1,3}=s_{2,1,3}=s_{1,2,3}=s_{2,3,3}=-\frac{\Delta z}{4}, \\
& s_{3,4,3}=s_{4,4,3}=s_{3,2,3}=s_{4,3,3}=\frac{\Delta z}{4} .
\end{aligned}
$$

a)

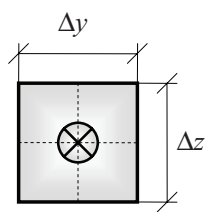

b)

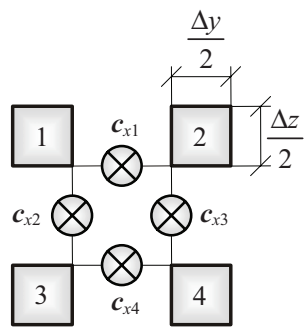

c)

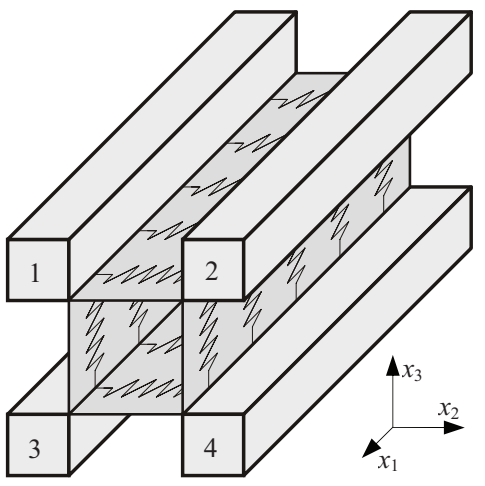

Fig. 4. Discrete model of beam: a) primary division, $b, c$ ) secondary division 
The matrices of boundary conditions for the system divided into four prisms have the form:

$$
\boldsymbol{M}=\left[\begin{array}{ll}
\boldsymbol{I}_{12 \times 12} & \mathbf{0}_{12 \times 12} \\
\mathbf{0}_{12 \times 12} & \mathbf{0}_{12 \times 12}
\end{array}\right], \quad \boldsymbol{N}=\left[\begin{array}{ll}
\mathbf{0}_{12 \times 12} & \mathbf{0}_{12 \times 12} \\
\boldsymbol{I}_{12 \times 12} & \mathbf{0}_{12 \times 12}
\end{array}\right] .
$$

For the discrete-continuous model prepared in the above way, the frequency characteristics were calculated (Fig. 5) for the harmonic force excitation with amplitude equal to one and acting at prism No. 2 , at point $\xi=0.1 \mathrm{~m}$. The displacement output signal was observed at prism No. 4 , at the $x=0.4 \mathrm{~m}$ point. The obtained characteristics were compared with those developed by using the finite element method and the ANSYS system (Fig. 5). The calculated natural frequencies are given in Table 1.

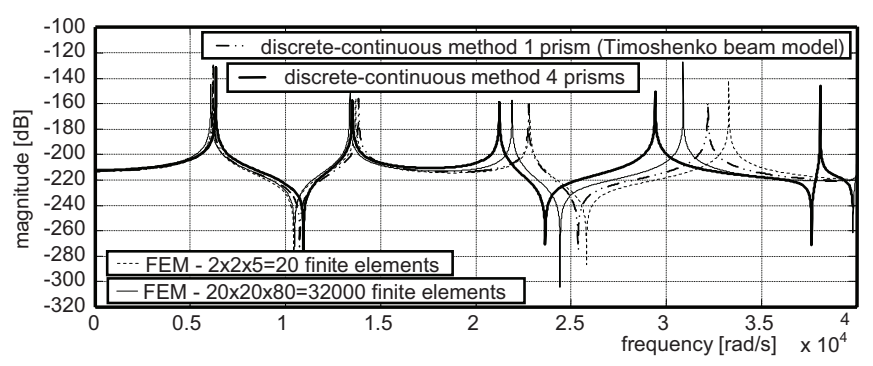

Fig. 5. Frequency characteristics

Table 1. Natural frequencies of the analysed beam [ $\mathrm{rad} / \mathrm{s}]$

\begin{tabular}{|c|c|c|c|c|c|c|c|}
\hline \multirow{2}{*}{ No. } & \multicolumn{2}{|c|}{ Proposed method } & \multicolumn{2}{|c|}{ FEM model } & \multicolumn{3}{|c|}{$\begin{array}{r}\text { Error with reference to FEM model } \\
20^{\prime} 20^{\prime} 80 \text { elem. [\%] }\end{array}$} \\
\cline { 2 - 8 } & 1 prism & 4 prisms & $\begin{array}{r}2 \times 2 \times 5 \\
(20 \text { el.) }\end{array}$ & $\begin{array}{r}20 \times 20 \times 80 \\
(32000 \text { el. })\end{array}$ & 1 prism & 4 prisms & $2 \times 2 \times 5$ \\
\hline 1 & 6211.15 & 6191.7 & 6164.68 & 6080.93 & 2.14 & 1.8 & 1.5 \\
\hline 2 & 13848.36 & 13568.31 & 13682.26 & 13381.3 & 3.5 & 1.4 & 2.2 \\
\hline 3 & 22763.9 & 21184.5 & 22817.3 & 21901.29 & 4 & 3.2 & 4.1 \\
\hline 4 & 32186.47 & 29587.12 & 33288.92 & 30888.13 & 4.2 & 4.2 & 7.7 \\
\hline 5 & 41982.17 & 38913.2 & 42763.4 & 40216.78 & 4.4 & 3.2 & 6.3 \\
\hline 6 & 47172.88 & 41321.6 & 45807.18 & 43225.17 & 9.1 & 4.4 & 5.9 \\
\hline
\end{tabular}

The discrete FEM model used as the reference consist of 32000 twenty-node hexahedron elements.

The characteristics in Fig. 5 show that the first two frequencies of the proposed discrete-continuous model, consisting of 4 prisms, and the reference FEM model with 32000 elements are very similar and differ by about 1.8 and $1.4 \%$ respectively (Table 1 ). In turn, the corresponding frequencies of the FEM model consisting of 20 finite elements differ respectively by about 1.5 and $2.2 \%$.

While the first frequency of the discrete-continuous model is slightly higher than the corresponding frequency of the FEM model with 20 elements, the second frequency is much lower and closer to the frequency of the reference FEM model (32000 elements). As the frequency grows, the discrete-continuous model appears to be more precise than the 20- element FEM model, although it consists only of four prisms.

The model consisting of one prism, which is equivalent to the Timoshenko beam model, turns out to be comparable with the 20 element FEM model. Although the first two frequencies of the Timoshenko beam model are less accurate, the next three frequencies are more accurate. But both these models are not accurate enough.

When the equivalent diameter $d$ of the beam is greater than $l /(2 \sqrt{\pi})$, we should use a higher-density mesh or, for example, the here proposed discrete-continuous method of modelling.

\section{SECOND EXAMPLE OF METHOD APPLICATION}

As the second illustrative example, let us consider a simply supported plate along its outline (Fig. 6a) with the following data: $E=2 \cdot 10^{11} \mathrm{~Pa}, G=8 \cdot 10^{10} \mathrm{~Pa}, \rho=8 \cdot 10^{10} \mathrm{~kg} / \mathrm{m}^{3}, v=0.25$, $\kappa=1, a=1 \mathrm{~m}, b=0.6 \mathrm{~m}, h=0.03 \mathrm{~m}$.

Comparative calculations were performed after implementing the proposed discrete-continuous method in the MATHEMATICA system and using the finite element method in the ANSYS system. The calculations were made for different numbers of prisms and finite elements. A sample discrete model of plate divided into twelve prisms is shown in Fig. 6. All prisms are connected via spring layers with distributed parameters. The stiffness coefficients of these layers were calculated from the following equations (25):

$c_{x 12}=\frac{E \Delta z}{\Delta y}, c_{x 13}=\frac{\kappa G \Delta z}{\Delta y}, c_{x 14}=\frac{E \Delta z^{3}}{12 \Delta y}, c_{x 15}=\frac{G \Delta z^{3}}{12 \Delta y}$

and are the same for spring layers from $c_{x 1}$ to $c_{x 11}$. For the supports along the plate outline, the stiffness coefficients were calculated using the following formulas: $c_{x 02}=5 \cdot c_{x 12}$, $c_{x 03}=5 \cdot c_{x 13}, c_{x 04}=0, c_{x 05}=0$.

Each prism has three degrees of freedom, which are: translational displacement along the $x_{3}$-axis and angular displacements around the $x_{1}$ and $x_{2}$-axes.

a)

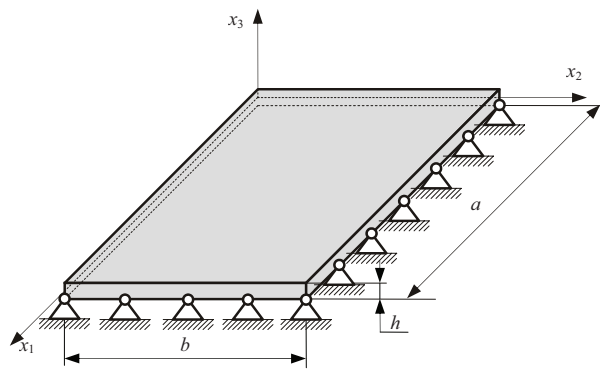

b)

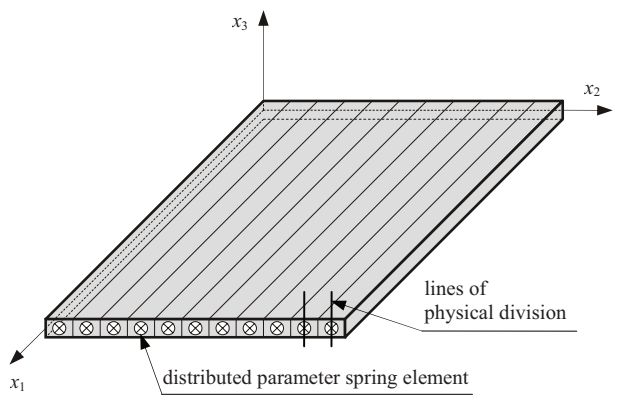


c)

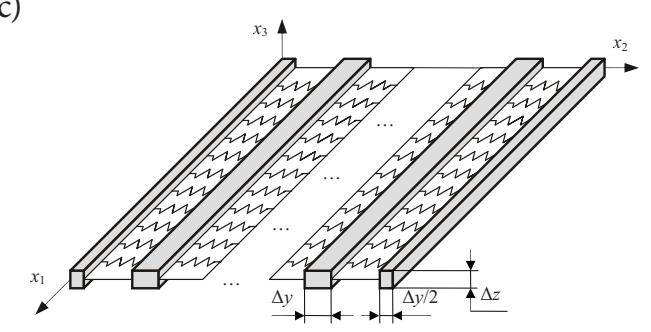

d)

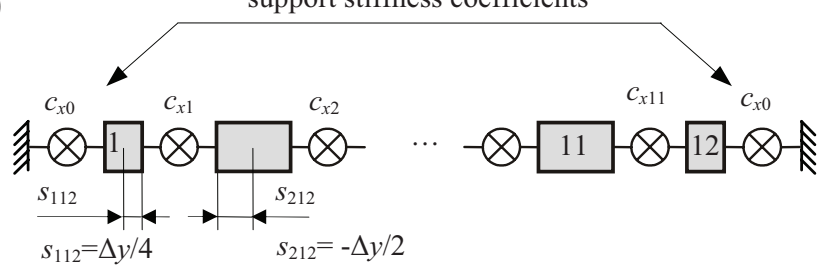

Fig. 6. The analysed plate: a) general scheme, b) primary virtual division, c) secondary physical division, d) equivalent scheme

The frequency characteristics were calculated for the harmonic force excitation having the amplitude equal to one and acting at the point with coordinates: $x_{1}=0.4 \mathrm{~m}$ and $x_{2}=0.2 \mathrm{~m}$. The displacement output signal was observed at the same point. The received characteristics were compared with those obtained by using the finite element method and the ANSYS system, and also with the exact frequencies calculated from the relation

$\omega(i, j)=\pi^{2}\left[\left(\frac{i}{a}\right)^{2}+\left(\frac{j}{b}\right)^{2}\right] \sqrt{\frac{E \cdot h^{3}}{12\left(1-v^{2}\right) \rho h}}$.

All frequency values are compared in Table 2.

a)

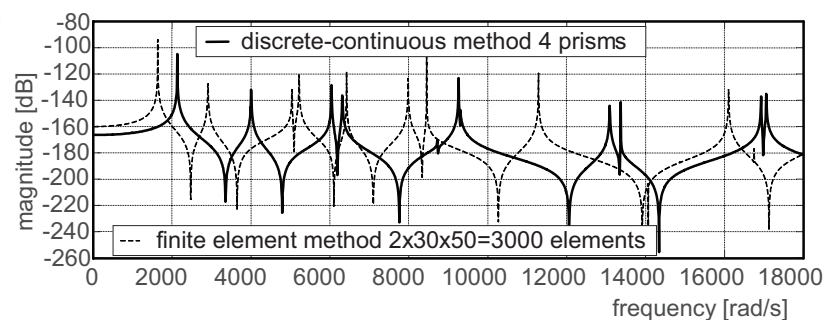

b)

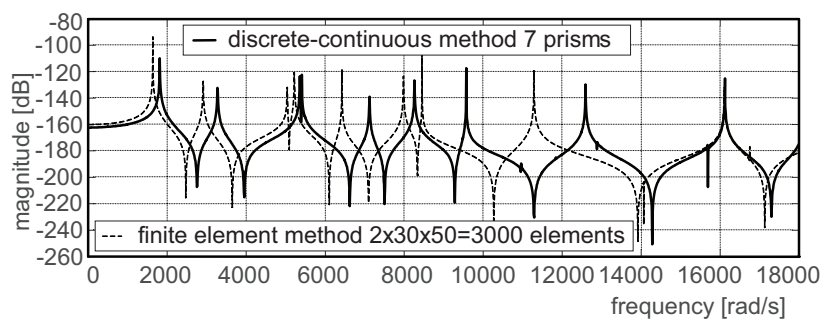

c)

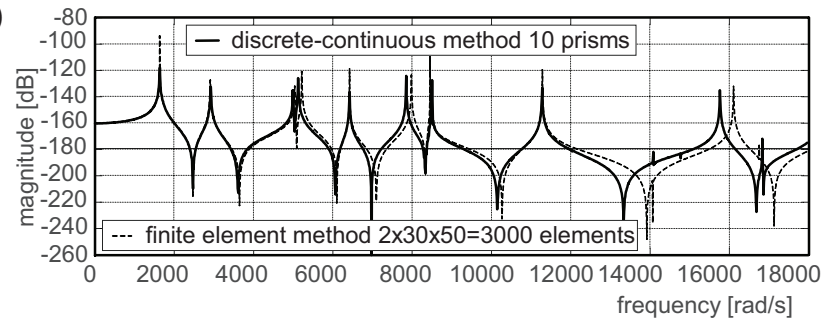

d)

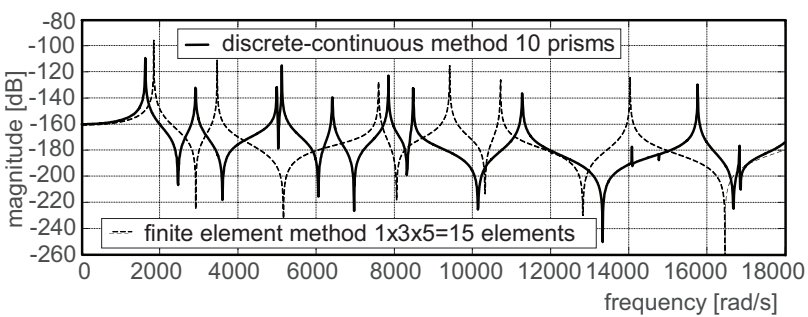

Fig. 7. Frequency characteristics

Table 2. Comparison of natural frequencies

\begin{tabular}{|c|c|c|c|c|c|c|c|c|}
\hline \multirow{2}{*}{$\begin{array}{c}\text { Mode } \\
\text { No. }\end{array}$} & \multirow{2}{*}{$\begin{array}{c}\text { Exact } \\
\text { frequency } \\
(57)\end{array}$} & \multicolumn{3}{|c|}{ Proposed method } & \multicolumn{3}{c|}{ FEM } & \multicolumn{2}{c|}{$\begin{array}{c}\text { Error in reference } \\
\text { to FEM model } \\
(3000 \text { elem. })\end{array}$} \\
\cline { 3 - 11 } & 4 prisms & 7 prisms & 10 prisms & $\begin{array}{c}1 \times 3 \times 5 \\
(15 \text { el. })\end{array}$ & $\begin{array}{c}2 \times 30 \times 50 \\
(3000 \text { el. })\end{array}$ & 10 prisms & $\begin{array}{c}1 \times 3 \times \times 5 \\
(15 \text { el. })\end{array}$ \\
\hline 1 & 1692 & 2130.35 & 1812.36 & 1632.08 & 1847.63 & 1633.88 & 0.1 & 13.08 \\
\hline 2 & 3036 & 4003.84 & 3274.93 & 2916.00 & 3468.88 & 2907.732 & 0.28 & 19.29 \\
\hline 3 & 5276 & 6042.83 & 5359.35 & 4996.67 & 7598.88 & 5039.429 & 0.84 & 50.78 \\
\hline 4 & 5425 & 6314.78 & 5411.67 & 5121.91 & 9417.23 & 5219.819 & 1.87 & 80.41 \\
\hline 5 & 6769 & 8727.93 & 7117.69 & 6417.73 & 10717.23 & 6427.07 & 0.14 & 66.75 \\
\hline 6 & 8412 & 9259.70 & 8263.06 & 7850.63 & 14031.61 & 7987.185 & 1.7 & 75.67 \\
\hline 7 & 9009 & 9305.27 & 9571.59 & 8487.07 & 19156.8 & 8459.681 & 0.32 & 126.44 \\
\hline 8 & 11648 & 12852.87 & 10958.05 & 10754.84 & 23491.57 & 11017.57 & 2.38 & 113.21 \\
\hline
\end{tabular}

The obtained results prove that the proposed method is effective and more accurate than the finite element method. Figure $7 \mathrm{~d}$ and Table 2 show that for a smaller number of elements (10 prisms) the presented method gives results closer to the accurate values than the finite element method with a larger number of elements (15 elements). The relative error of the proposed method with respect to the reference finite element method model with 3000 elements is smaller than that of the finite element model with a large number of elements (15 elements), Table 2.

\section{CONCLUSIONS}

The paper presents a hybrid discrete-continuous method of modelling. For the proposed approach, general partial differential equations were derived. These equations were verified and then written in a formalized matrix form, which is very convenient in application to numerical computations. A beam fixed at both ends and a simply supported plate were used to illustrate the general concept. The proposed method is described in a general form and can be also easily applied to other cases, not presented in this paper, including simply supported or one side fixed beams, as well as rectangular plates with different boundary conditions.

The performed numerical calculations and computer simulations showed that the proposed method, especially in the higher frequency range, gives more accurate results than the finite element method with the same or similar number of elements. In addition, even at a small number of elements, it gives a very good model accuracy and high convergence of numerical calculation results. The proposed method has been proved to be efficient and applicable to discrete-continuous modelling of dynamic systems. It can be applied especially to the analysis, simulation and numerical calculations of 
nontypical elements or systems requiring high accuracy. Semi-analytical solutions make the proposed method more accurate than the finite element method, especially in predicting high-frequency dynamics.

\section{BIBLIOGRAPHY}

1. Hein R., Orlikowski C.: Hybrid reduced model of rotor, The Archive of Mechanical Engineering, Vol. LX, No 3, pp. 319-333, 2013.

2. Hein R., Orlikowski C.: Optimum control of gyroscopic systems, Solid State Phenomena, Vol. 164, pp. 121-126, 2013.

3. Kaliński K. J., Galewski M. A.: A modified method of vibration surveillance by using the optimal control at energy performance index. Mechanical Systems and Signal Processing 58-59 (2015) 41-52.

4. Kaliński K. J., Galewski M. A.: Chatter vibration surveillance by the optimal-linear spindle speed control. Mechanical Systems and Signal Processing Volume 25, Issue 1, January 2011, Pages 383-399.

5. Kruszewski J., Gawroński W., Wittbrodt E., Najbar F., Grabowski S.: Metoda sztywnych elementów skończonych [Rigid finite element method], Arkady, Warszawa 1975.

6. Kujawa M., Szymczak C.: Numerical and experimental investigation of rotational stiffness of zed-purlins connection with sandwich panels// THIN-WALLED STRUCTURES. -Vol. 75, (2014), s.43-52.

7. Lipiński K.: Modeling and control of a redundantly actuated variable mass $3 \mathrm{RRR}$ planar manipulator controlled by a model-based feedforward and a model-based-proportional-derivative feedforward-feedback controller. Mechatronics 37 (2016), 42-53.

8. Orlikowski C., Hein R.: A simplified model of 3-D pipe system conveying flowing liquid, Solid State Phenomena, Vol. 198, pp. 621-626, 2013.

9. Orlikowski C., Hein R.: Modelling of geared multi-rotor system, Solid State Phenomena, Vol. 198, pp. 669-674, 2013.

10. Orlikowski C., Hein R.: Modelling and analysis of beam/ bar structure by application of bond graphs. Journal of Theoretical and Applied Mechanics, Vol. 49, No. 4, 2011.

11. Orlikowski C., Hein R.: Modal reduction and analysis of gyroscopic systems, Solid State Phenomena, Vol. 164, pp. 189-194, 2010.
12. Park D.-H., Yang B.: Distributed transfer function analysis of multi-body prismatic elastic solids, Int. J. of Structural stability and dynamics, Vol. 1, No. 1, pp. 83-104, 2001.

13. Wittbrodt E., Adamiec-Wójcik I., Wojciech S.: Dynamics of Flexible Multibody Systems. Rigid Finite Element Method. Foundations of Engineering Mechanics. Springer, Germany 2006. Pp. 225 (ISBN 3-540-32351-1, SPIN 11593553).

14. Wittbrodt E., Szczotka M., Maczyński A., Wojciech S.: Rigid finite element method in analysis of dynamics of offshore structures. Ocean Engineering \& Oceanography 1. Springer-Verlag Berlin Heidelberg, 2013. Pp. 252 (ISBN 978-3-642-29885-1, ISSN 2194-6396).

15. Yang B., Tan C.A.: Transfer functions of one-dimensional distributed parameter systems, ASME Journal of Applied Mechanics, Vol. 59, December, pp. 1009 - 1014 , 1992.

16. Yang B.: Distributed transfer function analysis of complex distributed parameter systems, ASME Journal of Applied Mechanics, Vol. 61, pp. 84 - 92, 1994.

17. Yang B., Zhou J.: Semi-analytical solution of 2-D elasticity problems by the strip distributed transfer function method, Int. J. Solids Structures, Vol. 33, No. 27, pp. 3983-4005,1996.

18. Zhou J., Feng Z.: Transient response analysis of onedimensional distributed parameter systems, Int. J. of Solids and structures, Vol. 36, pp. 2807-2824, 1999.

\section{CONTACT WITH THE AUTHOR}

\author{
Rafał Hein \\ e-mail:rahe@pg.gda.pl \\ Gdansk University of Technology \\ Narutowicza 11/12, 80-233 Gdansk \\ Poland
}

